Artigo Original

\title{
Percepção subjetiva do esforço, resposta afetiva e hipotensão pós-exercício em sessão de Tai Chi Chuan
}

\author{
Cheng Hsin Nery Chao ${ }^{1}$ \\ Alexandre Hideki Okano ${ }^{1}$ \\ Paulo André Holanda Savir ${ }^{1}$ \\ Emanuel Augusto Alves ${ }^{1}$ \\ Hassan Mohamed Elsangedy \\ Edilson Serpeloni Cyrino ${ }^{2}$ \\ Luiz Fernando de Farias Junior ${ }^{1}$ \\ Eduardo Caldas Costa ${ }^{1}$ \\ ${ }^{1}$ Universidade Federal do Rio Grande do Norte, Centro de Ciências da Saúde, Departamento de \\ Educação Física, Natal, RN, Brasil \\ ${ }^{2}$ Universidade Estadual de Londrina, Centro de Educação Física e Esporte, Departamento de \\ Educação Física, Londrina, PR, Brasil
}

\begin{abstract}
Resumo: O objetivo do estudo foi analisar a percepção subjetiva do esforço (PSE), resposta afetiva (RA) e pressão arterial pós-exercício em sessão aguda de Tai Chi Chuan (TCC). Vinte e quatro mulheres (47-79 anos; 9 normotensas e 15 hipertensas) foram submetidas a uma sessão de TCC e uma sessão controle (delineamento cruzado). A PSE foi avaliada pela escala de Borg (CR10), a RA pela escala de valência afetiva (+5/-5) e a pressão arterial (PA) pelo método oscilométrico (5, 10, 25, 20, 25 e 30 min pós-sessão). Redução significante da PA sistólica foi identificada aos 20 e 25 min após a sessão de TCC somente nas hipertensas. Os valores de RA e PSE foram $+2,8 \pm 1,9$ e 3,5 $\pm 1,2$, respectivamente. Os resultados sugerem que uma sessão de TCC de intensidade moderada foi prazerosa para as voluntárias (hipertensas e normotensas), embora tenha induzido discreto efeito hipotensor agudo na PA sistólica somente das hipertensas.
\end{abstract}

Palavras-chave: Saúde da mulher. Pressão arterial. Prevenção primária. Atividade física.

Rating of perceived exertion, affective response and post-exercise hypotension in Tai Chi Chuan session

\begin{abstract}
The aim of the study was to analyze the rating of perceived exertion (RPE), affective response (AR), and blood pressure (BP) post-exercise in acute Tai Chi Chuan (TCC) session. Twenty-four women (47-79 years; 9 normotensive and 15 hypertensive) performed one TCC and one control session (cross-over design). The RPE was assessed using the Borg's scale (CR10), the AR was assessed by the Feeling Scale $(+5 /-5)$ and the BP by the oscillometric method $(5,10,15,20,25$, and $30 \mathrm{~min}$ post-TCC session). A significant systolic BP reduction was observed in the 20 and $25 \mathrm{~min}$ post-TCC session only in the hypertensive women. The AR and RPE values were $+2.8 \pm 1.9$ e $3.5 \pm 1.2$, respectively. The results suggest that a moderate TCC exercise bout was pleasurable for the volunteers (normotensive and hypertensive), although it has induced a slight acute hypotensive effect on the systolic BP only in the hypertensive women.
\end{abstract}

Keywords: Women's health. Blood pressure. Primary prevention. Physical activity.

\section{Introdução}

A hipertensão arterial sistêmica (HAS) é uma doença crônico-degenerativa com destacada prevalência e repercussão sobre a saúde pública, uma vez que as doenças cardiovasculares (DCV), principal causa de morte no Brasil, está associada de forma independente, linear e contínua com o aumento da pressão arterial (PA) (SHIMOMURA; WAKABAYASHI, 2012; SOCIEDADE BRASILEIRA DE CARDIOLOGIA, 2010).

Assim, a prática regular de atividade física tem sido recomendada para a prevenção e tratamento da HAS (PESCATELLO et al., 2004; SOCIEDADE
BRASILEIRA DE CARDIOLOGIA, 2010), visto que o importante papel do exercício físico para a redução da PA tem sido demonstrado, tanto de forma crônica (CORNELISSEN; FARGARD, 2005; FAGARD; CORNELISSEN, 2007), a partir de treinamento físico sistematizado, quanto de forma aguda, após uma única sessão de exercício físico, em um fenômeno denominado de hipotensão pós-exercício (HPE) (HALLIWILL, 2001).

Nos últimos anos, a importância clínica da HPE tem atraído a atenção da comunidade científica, uma vez que existem indicativos da 
existência de uma importante relação entre HPE (efeito agudo) e adaptações hipotensoras em longo prazo (efeito crônico) (ANUNCIAÇÃO; POLITO, 2011; HAMER, 2006; ROSSI et al., 2012). Vale destacar que a HPE ocorre, principalmente, após sessões realizadas entre 3060 min sob intensidade moderada $(50-70 \%$ do consumo máximo de oxigênio $-\mathrm{VO}_{2} \mathrm{máx}$ ) (HALLIWILL, 2001).

Portanto, práticas corporais simples, de baixo custo e, principalmente, prazerosas e que possam favorecer $\mathrm{o}$ aumento da aderência em longo prazo devem ser estimuladas para a prevenção e tratamento da HAS. Para avaliar a sensação de prazer/desprazer durante a atividade física, Hardy e Rejeski (1989) propuseram uma escala subjetiva para avaliação da resposta afetiva (sensação), podendo esta variar entre os pólos "muito bom" e "muito ruim". Adicionalmente, de acordo com a teoria do modo duplo (EKKEKAKIS, 2003), há uma relação inversa entre a intensidade do exercício, mensurada tanto por meio de variáveis fisiológicas (p. ex., $\mathrm{FC}, \mathrm{VO}_{2}$ e ventilação pulmonar) quanto perceptuais (p. ex., PSE) e a sensação de prazer durante o exercício aeróbio. Em termos práticos, quanto maior o valor de PSE reportado pelo sujeito, menor a sua sensação de prazer.

Dessa forma, atividades físicas e/ou exercícios percebidos como prazerosos têm maior potencial para a manutenção da aderência em longo prazo (WILLIAMS et al., 2008). Considerando que o Tai Chi Chuan é uma modalidade de exercício físico predominantemente aeróbia, cuja intensidade da sessão de treinamento varia entre $50-60 \%$ do $\mathrm{VO}_{2}$ máx ("moderada"), independente da faixa etária (entre 25-80 anos) (LAN et al., 2001, 2004, 2008), é provável que uma sessão de Tai Chi Chuan possa ser considerada prazerosa e promova uma redução aguda dos valores pressóricos dos praticantes. Assim, o objetivo do presente estudo foi analisar o comportamento da PSE e da resposta afetiva durante uma aula de Tai Chi Chuan, assim como a PA pós-exercício.

\section{Sujeitos}

\section{Métodos}

Participaram do estudo 24 mulheres, nove normotensas e 15 hipertensas (47 e 79 anos), todas participantes de um programa de atividade física composto por práticas corporais orientais, promovido pelo Departamento de Educação Física, da Universidade Federal do Rio Grande do
Norte. Essas voluntárias foram selecionadas para este estudo, a partir dos seguintes critérios de inclusão: a) estar participando do programa há, pelo menos, quatro semanas e b) apresentar atestado médico liberatório para prática de atividade física. Todas as voluntárias após serem informadas sobre os procedimentos aos quais seriam submetidas, bem como sobre os possíveis riscos e benefícios da presente investigação, assinaram um termo de consentimento livre e esclarecido. Este estudo foi aprovado pelo Comitê de Ética em Pesquisa da Universidade Federal do Rio Grande do Norte (Processo 283/2010).

\section{Desenho experimental}

Inicialmente, as participantes foram questionadas sobre a utilização ou não de medicamentos para HAS e, na sequência, submetidas a uma avaliação antropométrica, para medida da massa corporal e estatura. A partir daí, três sessões preparatórias foram executadas ao longo de uma semana para familiarização das participantes aos instrumentos e procedimentos a serem empregados no estudo. Em cada uma dessas sessões as voluntárias receberam orientações para execução adequada do Tai Chi Chuan, utilização das escalas de Borg CR10 (BORG, 1982) e de valência afetiva (HARDY; REJESKI, 1989). Na semana seguinte às sessões preparatórias, as participantes foram submetidas a duas situações experimentais, a saber: a) sessão de Tai Chi Chuan e b) sessão controle (sem exercício). As duas sessões foram executadas de forma aleatória, em delineamento cruzado, em um intervalo de uma semana entre cada sessão. A sessão de Tai Chi Chuan apresentou duração de 60 min e foi composta por exercícios de alongamento e aquecimento para os grandes grupos musculares (cinco minutos); técnicas específicas da modalidade (50 min); e c) volta à calma (cinco minutos). Na sessão controle, as participantes permaneceram em repouso, na posição sentada, por $60 \mathrm{~min}$. A descrição das medidas empregadas em repouso e no pósexercício e na sessão controle são apresentadas a seguir.

\section{Respostas afetivas durante exercício}

O monitoramento do prazer ou desprazer durante a aula de Tai Chi Chuan foi realizado por meio da aplicação da escala de valência afetiva (VA), proposta por Hardy e Rejeski (1989). A escala é quantificada de +5 a -5 , correspondendo, respectivamente, aos dois descritores 
antagônicos do sentimento durante a atividade física: "muito bom" e "muito ruim". Além destes, a escala de VA apresenta descritores intermediários: $+3=$ bom; $+1=$ razoavelmente bom; 0 = neutro; -1 = razoavelmente ruim; $-3=$ ruim. Durante a sessão de Tai Chi Chuan a escala de VA foi respondida pelas voluntárias a cada $10 \mathrm{~min}$, sendo registrada a média aritmética de toda a aula.

\section{Monitoramento da PSE da sessão}

A quantificação da intensidade da aula de Tai Chi Chuan foi determinada pelo método da PSE da sessão (FOSTER et al., 2001). Para tanto, foi adotada a seguinte questão: "Como foi a sua sessão de treino?". A resposta foi fornecida, como recomendado, 30 min após o término do treino, a partir de uma escala adaptada de Borg CR10 (BORG, 1982). As participantes foram orientadas para escolher um descritor e depois um número de 0 a 10 , podendo este ser fornecido em decimais (por exemplo: 3,5). A escala utilizada apresentava os seguintes descritores: $0=$ repouso; 1 = muito, muito fácil; $2=$ fácil; $3=$ moderado; $4=$ um pouco difícil; $5=$ difícil; $7=$ muito difícil; 10 = máximo. $O$ valor máximo (10) deveria ser comparado ao maior esforço físico experimentado pela pessoa e o valor mínimo a condição de repouso absoluto (0).

\section{Medidas da pressão arterial}

Medidas de PA foram executadas antes do esforço, após 10 minutos de repouso (posição sentada) e nos minutos 5, 10, 15, 20, 25 e 30, após 0 encerramento de cada sessão experimental (controle e Tai Chi Chuan), de acordo com as recomendações da VI Diretrizes Brasileiras de HAS (SOCIEDADE BRASILEIRA DE CARDIOLOGIA, 2010). Dessa forma, três aferições da PA com intervalo de um minuto entre elas foram realizadas por meio de um equipamento automático da marca Omron $^{\circledR}$, modelo HEM-781, sendo registrada a média entre as duas últimas medidas como valor de referência.

\section{Análise estatística}

A normalidade na distribuição dos dados foi confirmada pelo teste de Shapiro-Wilk e a homocedasticidade foi identificada em todas as análises pelo teste de Levene. A esfericidade dos dados foi verificada por meio do teste de Mauchly, seguida pela correção de Greenhouse-Geisser quando os pressuspostos foram violados. Os resultados foram apresentados em valores de média e desvio-padrão. Para análise das diferenças entre a PA sistólica (PAS) e diastólica (PAD) em repouso e nos diferentes intervalos de tempo após as situações experimentais $(5,10,15$, 20, 25 e $30 \mathrm{~min}$ ) foi utilizada análise de variância (ANOVA) para medidas repetidas, seguida pelo teste post-hoc de Tukey para identificação das possíveis diferenças. O coeficiente de Pearson foi utilizado para análise da correlação entre PSE e $V A$, enquanto $o$ teste $t$ para amostras independentes foi adotado para as comparações entre os grupos. Para todas as análises foi estabelecida uma significância estatística de $5 \%$. Os dados foram processados no pacote estatí́stico SPSS ${ }^{\circledR}$ versão 17.0.

\section{Resultados}

$\mathrm{Na}$ Tabela 1 são apresentadas as características gerais da amostra do estudo. Nenhuma diferença estatisticamente significante entre os grupos foi identificada para as variáveis idade, massa corporal, estatura e índice de massa corporal $(p>0,05)$.

Tabela 1. Característica da amostra do estudo $(n=24)$

\begin{tabular}{lcc}
\hline \multicolumn{1}{c}{ Variáveis } & Normotensas $(\mathbf{n}=\mathbf{9})$ & Hipertensas $(\mathbf{n}=\mathbf{1 5})$ \\
\hline Idade (anos) & $63,6 \pm 10,4$ & $62,9 \pm 6,5$ \\
Massa corporal $(\mathrm{kg})$ & $71,9 \pm 12,3$ & $64,3 \pm 11,3$ \\
Estatura $(\mathrm{m})$ & $1,54 \pm 0,07$ & $1,54 \pm 0,06$ \\
Índice de massa corporal $\left(\mathrm{kg} / \mathrm{m}^{2}\right)$ & $30,4 \pm 4,8$ & $26,8 \pm 3,9$ \\
\hline
\end{tabular}

O comportamento da PAS após as duas sessões experimentais é ilustrado na Figura 1 (A e C). Uma redução significante $(p<0,05)$ nos valores da PAS foi verificada após a aula de Tai Chi Chuan, somente nas voluntárias hipertensas. Um efeito do tempo $\left(F_{(1,6)}=2,795 ; p=0,02\right)$ e interação condição e tempo $\left(\mathrm{F}_{(1,6)}=2,755 ; p=\right.$ $0,02)$ foi encontrada, embora um efeito principal da condição não tenha sido identificado $\left(\mathrm{F}_{(1,6)}=\right.$ $2,509 ; p=0,15)$. Em relação ao momento préexercício $(121,3 \pm 10,5 \mathrm{mmHg})$, nos minutos 20 $(110,1 \pm 12,3 \mathrm{mmHg})$ e $25(113,2 \pm 10,8 \mathrm{mmHg})$ 
uma redução da PAS no grupo hipertenso foi observada. Para o grupo normotenso, não houve efeito principal do tempo $\left(\mathrm{F}_{(1,6)}=0,755 ; p=0,60\right)$ ou da condição $\left(F_{(1,6)}=0,151 ; p=0,70\right)$ e, consequente, da interação condição e tempo
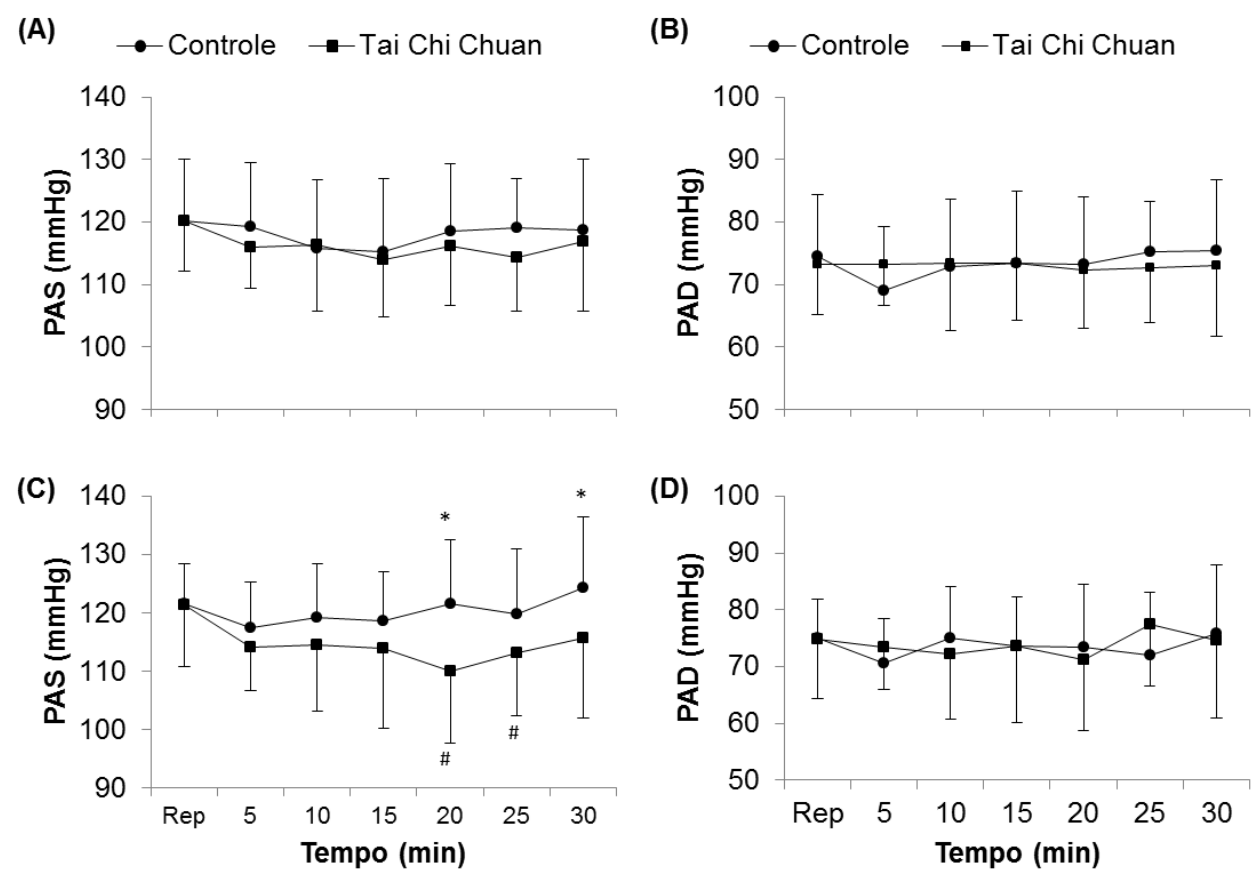

Figura 1. Comportamento da pressão arterial do grupo normotenso (Figuras $A$ e $B, n=9$ ) e hipertenso (Figuras $\mathrm{C}$ e $\mathrm{D}, \mathrm{n}=15)$. ${ }^{*} p<0,05$ vs.condição Tai Chi Chuan; ${ }^{\sharp} p<0,05$ vs. pré-exercício.

\section{(A)}

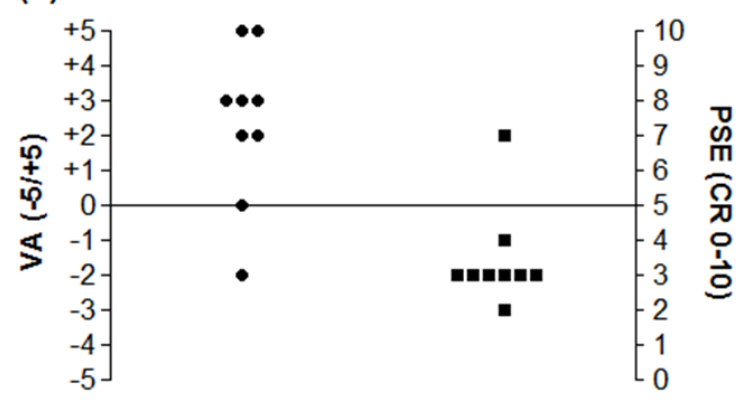

(B)

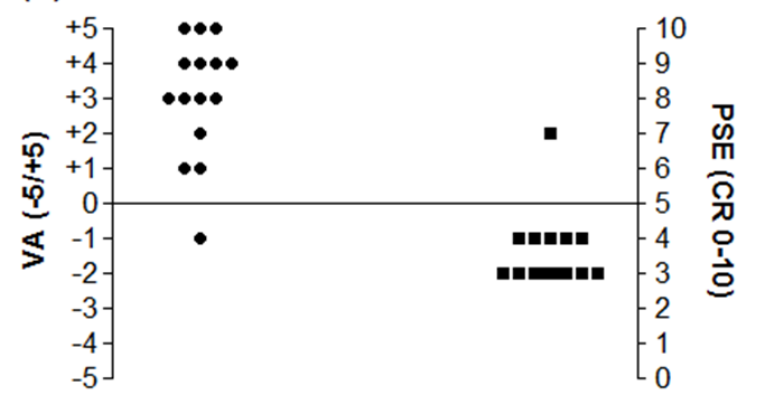

Figura 2. Comportamento individual da percepção subjetiva do esforço e valência afetiva no grupo normotenso (Figura $A, n=9$ ) e hipertenso (Figura B, $n=15$ ) durante a sessão de Tai Chi Chuan.

\section{Discussão}

Os principais achados do nosso estudo foram que a sessão de Tai Chi Chuan: a) caracterizouse como moderada no que se refere à intensidade; b) foi prazerosa para as participantes e c) apresentou discreto efeito hipotensor agudo sobre a PAS, somente no grupo de hipertensas. De acordo com as informações disponíveis na literatura até 0 presente momento, este parece ser o primeiro estudo com monitoramento da 
intensidade e sensação de prazer durante a prática do Tai Chi Chuan, além do registro da HPE induzido por esta modalidade de exercício físico.

As participantes hipertensas do nosso estudo, diferentemente das normotensas, apresentaram redução da PAS pós-sessão de TCC. Anteriormente, Harvey et al. (2005) já haviam encontrado um queda na PAS $(5 \mathrm{mmHg})$ e na PAD (6 mmHg) após uma sessão moderada de exercício aeróbio ( $45 \mathrm{~min}$ à $60 \%$ do $\mathrm{VO}_{2}$ máx) em mulheres normotensas pós-menopausadas. Adicionalmente, os autores observaram que as voluntárias com valores de PA média préexercício mais elevados exibiram maior queda pós-exercício, corroborando em parte com as informações encontradas neste estudo.

O efeito hipotensor agudo de uma sessão de exercício, embora seja temporário, é clinicamente importante no controle da PA (HALLIWILL et al., 2000). Pescatello et al. (2001) verificaram, mediante uma revisão sistemática composta por 23 estudos envolvendo hipertensos e normotensos, uma redução média de 3,2 e 1,8 $\mathrm{mmHg}$ na PAS e PAD, respectivamente, medida por monitorização ambulatorial da PA (MAPA), nas $24 \mathrm{~h}$ posteriores à uma sessão de exercício aeróbio. Vale ressaltar que essa redução média ocorreu, sobretudo, em virtude da queda substancial da PA nas primeiras horas após o fim da prática do exercício, o que reforça a importância clínica da HPE.

Mais recentemente, Anunciação e Polito (2011) conduziram uma revisão sobre HPE em indivíduos hipertensos e constataram que esse fenômeno tem sido observado de forma consistente, tanto para a PAS quanto PAD, após a prática de exercícios predominantemente aeróbios com intensidade entre $60-70 \%$ do $\mathrm{VO}_{2}$ máx e duração entre $30-45 \mathrm{~min}$ (semelhante a sessão de Tai Chi Chuan utilizada neste estudo). Entretanto, nenhum estudo revisado envolveu a prática do Tai Chi Chuan. Nossos dados corroboram em parte com os resultados de duas revisões disponíveis na literatura (ANUNCIACÃ̃O; POLITO, 2011; PESCATELLO et al. 2001), visto que uma redução significante na PAS ( 11-13 $\mathrm{mmHg}$ ) foi identificada somente no grupo hipertenso, após a sessão de Tai Chi Chuan.

Lan et al. (2001) avaliaram a intensidade de uma aula de Tai Chi Chuan em 15 indivíduos do sexo masculino (26-56 anos). Os autores revelaram que a sessão apresentou um caráter moderado, com os participantes se exercitando em média a $58 \%$ da $F C$ de reserva (FCR) e $55 \%$ do $\mathrm{VO}_{2}$ máx, além de apresentarem um concentração média de lactato na ordem de 3,8 $\mathrm{mmol} / \mathrm{l}$ após o fim da atividade. Mais recentemente, pesquisadores demonstraram que a intensidade do Tai Chi Chuan, independente do gênero, não é diferente entre jovens, indivíduos de meia idade e idosos, sendo de $\sim 55 \%$ e $\sim 50 \%$ da FCR para homens e mulheres, respectivamente (LAN et al., 2001, 2008). Esses achados parecem corroborar com os resultados do presente estudo, uma vez que $\sim 80 \%$ das voluntárias reportaram a PSE entre 3 e 4 , o que equivale a $40-59 \%$ do $\mathrm{VO}_{2}$ máx e $55-69 \%$ da $\mathrm{FC}$ máxima (intensidade moderada) (FLETCHER et al., 2001). Esse fato, provavelmente, tenha influenciado a HPE, visto que tal fenômeno ocorre, principalmente, após sessões de exercício com maior duração e intensidade moderada

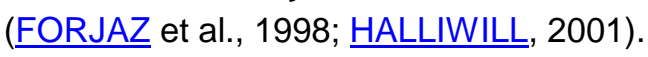

Com relação à resposta afetiva, a sessão de Tai Chi Chuan promoveu sensação de prazer na maioria das praticantes. Esses achados corroboram com a teoria do modo duplo (EKKEKAKIS, 2003; EKKEKAKIS; ACEVEDO, 2006), que presume que a resposta afetiva é determinada por fatores cognitivos (personalidade, auto-eficácia, determinação, etc.) e interoceptivos (metaboreceptores, baroceptores, termoceptores, nociceptores, etc.). A teoria de modo duplo assume que exercícios realizados na intensidade do domínio moderado (inferiores ao limiar anaeróbio - LA) promovem sensações de prazer, em virtude da reduzida perturbação dos sistemas orgânicos e a manutenção da homeostase celular. Em contrapartida, em esforços realizados no domínio intenso (correspondente ao LA), que aumentam as participações de processos cognitivos, observa-se alta variação interindividual na resposta afetiva, com alguns sujeitos interpretando a intensidade do esforço como prazerosa e outros indivíduos considerando-a desconfortável. Já na intensidade do domínio severo (acima do LA), cujas vias interoceptivas atuam de maneira determinante, a resposta afetiva é desprazerosa para a maioria dos indivíduos (EKKEKAKIS, 2003).

A correlação negativa entre PSE da sessão e VA encontrada em nosso estudo está de acordo 
com os pressupostos da teoria do modo duplo, pois os indivíduos que apresentaram maior PSE durante a aula de TCC reportaram menores valores na escala de VA, indicando que quanto maior o esforço percebido menor foi a sensação de prazer.

O conhecimento da resposta afetiva durante uma sessão de exercício tem papel importante na aderência aos programas de condicionamento físico voltados para saúde. A teoria hedônica da motivação (KAHNEMAN, 1999) sugere que quando uma atividade realizada pelo sujeito é percebida de forma prazerosa, provavelmente, será repetida pelo mesmo. Por outro lado, se a atividade acarreta sensação de desprazer, desconforto, dor e/ou exaustão, a probabilidade em repetir ou aderir àquela atividade é reduzida. Nesse sentido, Williams et al. (2008) demonstraram que participantes que apresentaram sensações de prazer durante uma única sessão de exercício físico, após um ano, aumentaram seus níveis de atividade física. Por outro lado, aqueles que perceberam a sessão de exercício físico como desprazerosa, diminuíram seus níveis de atividade física. Diante disso, nosso estudo demonstrou que o Tai Chi Chuan foi percebido pela maioria das participantes como uma atividade de intensidade moderada que promove sensações de prazer 0 que, possivelmente, poderá favorecer o aumento dos níveis de atividade física dos praticantes.

Além da possível contribuição para o aumento da aderência em programas de condicionamento físico voltados para a saúde, do ponto de vista da saúde cardiovascular, o Tai Chi Chuan parece apresentar potencial para ser utilizado como prática adjuvante na prevenção e tratamento da HAS, tendo em vista que recentes revisões sistemáticas têm apontado que essa prática oriental, assim como as atividades aeróbias clássicas (caminhada, corrida e ciclismo), apresenta efeitos positivos em longo prazo sobre a PA (LEE et al., 2007; ROGERS et al., 2009; YEH et al., 2008, 2009).

Apesar dos achados do presente estudo serem bastante promissores, algumas limitações metodológicas precisam ser destacadas, tais como a ausência de avaliação do nível de atividade física habitual das voluntárias e o tempo de prática do TCC, padronização das condições clínicas das voluntárias, ausência de registro da
FC durante a aula de TCC, além do tempo limitado de avaliação da PA pós-exercício.

Por fim, os resultados do nsso estudo sugerem que o Tai Chi Chuan é uma modalidade de atividade física prazerosa e de intensidade moderada quando aplicado em mulheres de meia idade e idosas. Além disso, uma única sessão dessa prática oriental pode apresentar discreto efeito hipotensor agudo, essencialmente na PAS em mulheres hipertensas. Estudos adicionais com utilização de medidas fisiológicas de monitoramento da intensidade do exercício ( $p$. ex., $\mathrm{FC}, \mathrm{VO}_{2}$ máx e lactato) se fazem necessários para confirmação desses achados. Além disso, um acompanhamento mais elaborado da PA, por meio de MAPA, pode auxiliar na compreensão da magnitude e a duração da HPE provocada por uma sessão de Tai Chi Chuan em diferentes populações.

\section{Referências}

ANUNCIAÇÃO, P. G.; POLITO, M. D. A review on post-exercise hypotension in hypertensive individuals. Arquivos Brasileiros de

Cardiologia, Rio de Janeiro, v. 96, n. 5, p. e100e109, 2011. DOI:

$<$ http://dx.doi.org/10.1590/S0066782X2011005000025>

BORG, G. A. Psychophysical bases of perceived exertion. Medicine and Science in Sports and Exercise, Hagerstown, v. 14, n. 5, p. 377-381, 1982.

CASONATTO, J.; POLITO, M. D. Hipotensão pósexercício aeróbio: uma revisão sistemática.

Revista Brasileira de Medicina do Esporte, Niterói, v. 15, n. 2, p. 151-157, 2009. DOI: $<$ http://dx.doi.org/10.1590/S151786922009000200014>

CORNELISSEN, V. A.; FAGARD, R. H. Effects of endurance training on blood pressure, blood pressure-regulating mechanisms, and cardiovascular risk factors. Hypertension, v. 46, n. 4, p. 667-675, 2005. DOI: $<$ http://dx.doi.org/10.1161/ 01.HYP.0000184225.05629.51>

EKKEKAKIS, P. Pleasure and displeasure from the body: perspective from exercise. Cognition \& Emotion, London, v. 17, n. 2, p. 213-239, 2003. DOI: <http://dx.doi.org/10.1080/02699930302292>

EKKEKAKIS, P.; ACEVEDO, E. O. Affective responses to acute exercise: toward a psychobiological dose-response model. In: ACEVEDO, E. O.; EKKEKAKIS, P., editors. 
Psychobiology of physical activity. Champaign: Human Kinetics, p. 91-109, 2006.

FAGARD, R. H.; CORNELISSEN, V. A. Effect of exercise on blood pressure control in hypertensive patients. European Journal of Cardiovascular Prevention and Rehabilitation, London, v. 14, n. 1, p. 12-17, 2007. DOI: <http://dx.doi.org/ 10.1097/HJR.0b013e3280128bbb>

FLETCHER, G. F.; BALADY, G. J.; AMSTERDAM, E. A.; CHAITMAN, B.; ECKEL, R.; FLEG, J.; FROELICHER, V. F.; LEON, A. S.; PIÑA, I. L.; RODNEY, R.; SIMONS-MORTON, D. A.; WILLIAMS, M. A.; BAZZARRE, T. Exercise standards for testing and training: a statement for healthcare professionals from the American Heart Association. Circulation, Hagerstown, v. 104, n. 14, p. 1694-1740. 2001. DOI: <http://dx.doi.org/10.1161/hc3901.095960>

FORJAZ, C. L. M.; SANTAELLA, D. F.; REZENDE, L. O.; BARRETTO, A. C. P.; NEGRÃO, C. E. A duração do exercício determina a magnitude e a duração da hipotensão pósexercício. Arquivos Brasileiros de Cardiologia, Rio de Janeiro, v. 70, n. 2, p. 99-104, 1998. DOI: $<$ http://dx.doi.org/10.1590/S0066782X1998000200006>

FOSTER, C.; FLORHAUG, J. A.; FRANKLIN, J.; GOTTSCHALL, L.; HROVATIN, L. A.; PARKER, S.; DOLESHAL, P.; DODGE, C. A new approach to monitoring exercise training. Journal of Strength and Conditioning Research, Champaign, v. 15, n. 1, p. 109-115, 2001.

HALLIWILL, J. R. Mechanisms and clinical implications of post-exercise hypotension in humans. Exercise and Sport Sciences Reviews, Hagerstown, v. 29, n. 2, p. 65-70, 2001.

HALLIWILL, J. R.; MINSON, C. T.; JOYNER, M. J. Effect of systemic nitric oxide synthase inhibition on postexercise hypotension in humans. Journal of Applied Physiology, Bethesda, v. 89, n. 5, p. 1830-1836, 2000.

HAMER, M. The anti-hypertensive effects of exercise: integrating acute and chronic mechanisms. Sports Medicine, Auckland, v. 36, n. 2, p. 109-116, 2006.

HARDY, C. J.; REJESKI, W. J. Not what, but how one feels: measurement of affect during exercise. Journal of Sport \& Exercise Psychology, Champaign, v. 11, n. 3, p. 304-317, 1989.

HARVEY, P. J.; MORRIS, B. L.; KUBO, T.; PICTON, P. E., SU, W. S.; NOTARIUS, C. F.; FLORAS, J. S. Hemodynamic after-effects of acute dynamic exercise in sedentary normotensive postmenopausal women. Journal of Hypertension, London, v. 23, n. 2, p. 285-292, 2005.

KAHNEMAN, D. Objective happiness. In: KAHNEMAN, D.; DIENER, E.; SCHWARZ, N., editors. Well-being: the foundation of hedonic psychology. New York: Russell Sage Foundation, p. 3-25, 1999.

KAUFMAN, F. L.; HUGHSON, R. L.; SCHAMAN, J. P. Effect of exercise on post-exercise blood pressure in normotensive and hypertensive subjects. Medicine and Science in Sports and Exercise, Hagerstown, v. 19, n. 1, p. 17-20, 1987.

LAN, C.; CHEN, S. Y.; LAI, J. S.; WONG, M. K. Heart rate responses and oxygen consumption during tai chi chuan practice. The American Journal of Chinese Medicine, Garden City, v. 29, n. 3-4, p. 403-410, 2001. DOI: <http://dx.doi.org/ 10.1142/S0192415X01000423>

LAN, C.; CHEN, S. Y.; LAI, J. S. Relative exercise intensity of tai chi chuan is similar in different ages and gender. The American Journal of Chinese Medicine, Garden City, v. 32, n. 1, p. 151-160, 2004. DOI:

<http://dx.doi.org/10.1142/S0192415X04001746>

LAN, C.; CHEN, S. Y.; LAI, J. S. The exercise intensity of tai chi chuan. Medicine in Sport Science, New York, v. 52, p. 12-19, 2008. DOI: <http://dx.doi.org/ 10.1159/000134225>

LEE, M. S.; PITTLER, M. H.; TAYLOR-PILIAE, R. E.; ERNST, E. Tai chi for cardiovascular disease and its risk factors: a systematic review. Journal of Hypertension, London, v. 25, n. 9, p. 19741975, 2007. DOI: <http://dx.doi.org/ $\underline{10.1097 / H J H .0 b 013 e 32828 c c 8 c d>}$

PESCATELLO, L. S.; FRANKLIN, B. A.; FAGARD, R.; FARQUHAR, W. B.; KELLEY, G. A. RAY, C. A. American College of Sports Medicine position stand: exercise and hypertension. Medicine and Science in Sports and Exercise, Hagerstown, v. 36, n. 3, p. 533-553, 2004.

PESCATELLO, L. S.; KULIKOWICH, J. M. The aftereffects of dynamic exercise on ambulatory blood pressure. Medicine and Science in Sports and Exercise, Hagerstown, v. 33, n. 11, p. 18551861, 2001.

ROGERS, C. E.; LARKEY, L. K.; KELLER, C. A review of clinical trials of tai chi and qigong in older adults. Western Journal of Nursing Research, Beverly Hills, v. 31, n. 2, p. 245-279, 2009. DOI: <http://dx.doi.org/ 10.1177/0193945908327529> 
ROSSI, A.; DIKAREVA, A.; BACON, S. L.; DASKALOPOULOU, S. S. The impact of physical activity on mortality in patients with high blood pressure: a systematic review. Journal of Hypertension, London, v. 30, n. 7, p. 1277-1288, 2012. DOI: <10.1097/HJH.0b013e3283544669>

RUECKERT, P. A.; SLANE, P. R.; LILLIS, D. L.; HANSON, P. Hemodynamic patterns and duration of post-dynamic exercise hypotension in hypertensive humans. Medicine and Science in Sports and Exercise, Hagerstown, v. 28, n. 1, p. 24-32, 1996.

\section{SOCIEDADE BRASILEIRA DE CARDIOLOGIA.}

VI Diretrizes Brasileiras de Hipertensão. Arquivos

Brasileiros de Cardiologia, Rio de Janeiro, v. 95, n. 1, supl. 1, p. 1-51, 2010. DOI:

$<$ http://dx.doi.org/10.1590/S0066-

782X2010001700001>

SHIMOMURA, T.; WAKABAYASHI, I.

Associations of cardiovascular risk factors with prehypertension and hypertension in women.

Blood Pressure, London, v. 21, n. 6, p. 345-351, 2012. DOI:

$<$ http://dx.doi.org/10.3109/08037051.2012.686177

$>$

WILLIAMS, D. M.; DUNSIGER, S.; CICCOLO, J. T.; LEWIS, B. A.; ALBRECHT, A. E.; MARCUS, B. $\mathrm{H}$. Acute affective response to a moderateintensity exercise stimulus predicts physical activity participation 6 and 12 months later.

Psychology of Sport and Exercise, New York, v. 9, n. 3, p. 231-245, 2008. DOI:

$<$ http://dx.doi.org/10.1016/j.psychsport.2007.04.00 2>

YEH, G. Y.; WANG, C.; WAYNE, P. M.;

PHILLIPS, R. S. The effect of tai chi exercise on blood pressure: a systematic review. Preventive Cardiology, Hoboken, v. 11, n. 2, p. 82-89, 2008. DOI: <http://dx.doi.org/10.1111/j.1751-

7141.2008.07565.x>

YEH, G. Y.; WANG, C.; WAYNE, P. M.; PHILLIPS, R. S. Tai chi exercise for patients with cardiovascular conditions and risk factors: a systematic review. Journal of Cardiopulmonary Rehabilitation and Prevention, Philadelphia, v. 29, n. 3, p. 152-160, 2009. DOI: $<$ http://dx.doi.org/10.1097/HCR.0b013e3181a3337 9>
Agradecimentos: Os autores agradecem a Coordenação de Aperfeiçoamento de Pessoal de Nível Superior (CAPES) pela bolsa de mestrado (L.F.F.J.) e ao Conselho Nacional de Desenvolvimento Científico e Tecnológico (CNPq) pelas bolsas de produtividade em pesquisa (A.H.O. e E.S.C.) outorgadas.

Endereço:

Eduardo Caldas Costa

Departamento de Educação Física - Centro de Ciências da Saúde - UFRN

Campus Universitário Lagoa Nova

Natal RN Brasil

59078-970

Telefone: (84) 32153459

e-mail: ecc.ufrn@hotmail.com

Recebido em: 5 de janeiro de 2011. Aceito em: 6 de fevereiro de 2013.

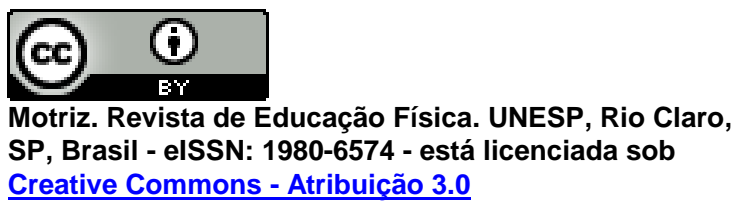
Creative Commons - Atribuição 3.0 